\title{
Natural language processing in mental health applications using non-clinical texts†
}

\author{
R A F A E L A. CA L V O ${ }^{1}$, D A V I D N. M I L N E ${ }^{1}$, \\ M. S A Z Z A D H U S A I N ${ }^{1,2}$ \\ and HELEN CHR ISTE N E N ${ }^{3}$ \\ ${ }^{1}$ School of Electrical and Information Engineering, The University of Sydney, Australia \\ e-mail: Rafael.calvo@sydney.edu.au, david.milne@sydney.edu.au \\ ${ }^{2}$ Health and Biosecurity, CSIRO, Epping, NSW, Australia or Health and Biosecurity, CSIRO, Australia \\ e-mail: sazzad.hussain@csiro.au \\ ${ }^{3}$ Black Dog Institute, University of New South Wales, Australia \\ e-mail: helen.christensen@unsw.edu.au
}

(Received 20 January 2016; revised 16 November 2016; accepted 17 November 2016;

first published online 30 January 2017)

\begin{abstract}
Natural language processing (NLP) techniques can be used to make inferences about peoples' mental states from what they write on Facebook, Twitter and other social media. These inferences can then be used to create online pathways to direct people to health information and assistance and also to generate personalized interventions. Regrettably, the computational methods used to collect, process and utilize online writing data, as well as the evaluations of these techniques, are still dispersed in the literature. This paper provides a taxonomy of data sources and techniques that have been used for mental health support and intervention. Specifically, we review how social media and other data sources have been used to detect emotions and identify people who may be in need of psychological assistance; the computational techniques used in labeling and diagnosis; and finally, we discuss ways to generate and personalize mental health interventions. The overarching aim of this scoping review is to highlight areas of research where NLP has been applied in the mental health literature and to help develop a common language that draws together the fields of mental health, human-computer interaction and NLP.
\end{abstract}

\section{Introduction}

People write to communicate with others. In addition to describing simple factual information, people also use writing to express their activities, and convey their feelings, mental states, hopes and desires. Recipients then use this written information from emails and other forms of social media texts to make inferences, such as what

\footnotetext{
${ }^{\dagger} \mathrm{RAC}$ and $\mathrm{SH}$ are supported by the Young and Well Cooperative Research Centre, which is established under the Australian Government's Cooperative Research Centres Program. RAC is supported by an Australian Research Council Future Fellowship FT140100824. RAC and DM are supported by an Australian Research Council Linkage Project. HC is supported by an NHMRC Fellowship 1056964.
} 
someone else is feeling, which in turn influences interpersonal communication. Even when writing is shaped by the way someone wants to be perceived, this text still provides important cues to help friends and family recognize important life events for them to respond to with support and encouragement.

When people write digitally (e.g. on email or social media), their texts are processed automatically. Natural language processing (NLP) techniques make inferences about what people say and feel, and these inferences can trigger messages or other actions. One of the most common uses of NLP is in the marketing sector where companies analyze emails and social media to generate targeted advertising and other forms of 'interventions' (generally aimed at changing our behavior toward buying something or following a link).

However, potential applications of NLP techniques extend beyond marketing. For example, NLP techniques have been identified as an important area of growth within the artificial intelligence (AI) in medicine community (Peek et al. 2015). In this paper, we discuss the potential for NLP techniques to be utilized in the mental health sector. Mental health applications are designed to support mental health and wellbeing in an online environment. These applications are reliant on interdisciplinary collaboration between researchers and practitioners from areas such as computational linguistics, human-computer interaction and mental health (and mental health service delivery). Interdisciplinary collaborations benefit from the development of a common language and body of research evidence (Calvo et al. 2016). Regrettably, there is a paucity of organized literature at this intersection between NLP, human-computer interaction and mental health research. This paper aims to help researchers in these areas envision and work toward new mental health applications. The paper is structured as follows:

- Section 2 provides a brief explanation of the methodology used to identify relevant literature, and the inclusion and exclusion procedures applied to this scoping study.

- Section 3 describes the types of textual data that has been the focus of research to date. In this section, the different data sources (ranging from lengthy diaries to brief tweets), and existing datasets that have been gathered and annotated (with moods, suicidal intent, etc.) using NLP are discussed.

- Section 4 describes the techniques that researchers have applied to make inferences or generate diagnoses about the emotional state or mental health of the authors of these texts. This work draws widely upon text classification and NLP.

- Section 5 identifies the different types of automated interventions for supporting mental health. These range from simple canned (i.e. the same for all users) interventions to personalized interventions. Where possible, we describe the specific studies in order to evaluate the reported efficacy of these interventions.

We conclude this section with a discussion of the gaps in the literature and future opportunities in this research domain. 


\section{Methods and overview}

Systematic reviews such as those published by the Cochrane Collaboration require an understanding of the existing literature and its gaps and involve a process that investigates a research question and develops and applies a framework for exploring a question utilizing existing literature (Armstrong et al. 2011). When an area is complex, very broad, or has not been reviewed before, a scoping review that maps the key concepts maybe more appropriate. Here, we aim to answer the following question: What Natural Language Technologies have been used with user generated data in the area of mental health? The multidisciplinary nature of this question, and the differences in terminology used across disciplines, means a systematic review methodology is not practical.

In the period between August 2014 and May 2015, the authors performed multiple searches using Google Scholar. Relevant research published after this period was added during the reviewing process. Google Scholar was chosen because it indexes journals, conferences and patent documents. Conference papers and patents are particularly important sources because a significant amount of the research in NLP and computer science is not published in journals, which is the standard in the mental health domain.

As is common in scoping reviews and when the review covers several research fields a definite set of search terms was not used. We performed multiple iterations of widening search terms and then identified and selected important and recurrent themes. In the first iteration, approximately 50 papers were identified. From this literature, three stages of research reporting emerged: Data, Labeling and Intervention. Inclusion criteria for each of these stages involved:

- In Data, we focus exclusively on textual data (as opposed to physiological signals, activity, etc.) that has been analyzed for mental health applications. We also focus on texts that have been written by users (e.g. mostly consumers, occasionally patients) rather than doctors or researchers. There is a significant amount of research focused on using NLP to process clinical notes, medical records and academic research papers, but they do not contribute to the focus of this review (user-generated texts) - for a review see Abbe et al. (2015). Initial search terms included the name of data sources often used in NLP projects (Twitter, Facebook, etc.) AND Mental Health AND NLP (written as Facebook + 'mental health' + 'NLP'). Google Scholar generally sorts papers by the number of citations so papers with few or no citations might have been missed. Links were followed and if found appropriate, added to a database. We run searches for (Facebook OR Twitter) AND (Mental Health) AND (NLP) in PubMed, but did not get any results. The search for Mental Health AND NLP gave 35 results. The references in highly relevant papers were scanned for title and authors that seemed relevant. This process was followed for each of the three areas (i.e. stages) by one of the authors. In later iterations, all authors were involved.

- In Automated Labeling, we focus on applications of NLP to the textual data collected in the previous section. We searched for papers that used the 
different components of a classification system: feature extraction, feature selection and classification and mental health and NLP. There is an extensive machine learning literature on document classification techniques that has been reviewed previously (Sebastiani 2002; Kotsiantis 2007). For the purposes of this review, only those studies that included a mental health application were considered within the scope of the present discussion.

- In Intervention, we considered studies that showed ways in which NLP could be used in computer interventions to support mental health. Hypothetical uses of NLP in psycho-education are discussed but we excluded those psychoeducation interventions where texts or multimedia are presented to clients without personalization or where they did not utilize NLP. Furthermore, while we have focused on mental health interventions, because of the scarcity of literature in this area, we extended the scope of the literature search to include publications from other health areas that highlight novel uses of NLP that could also be applied as a mental health intervention.

Some resources, such as conference proceedings and literature reviews were useful to bootstrap our literature search. These include the First Workshop on Computational Linguistics and Clinical Psychology (Resnik, Resnik and Mitchell 2014) held within the annual meeting of the American Association on Computational Linguistics, the leading NLP conference. The papers published in the proceedings from this conference highlight the breath of current topics being considered: depression, Alzheimer's, autism, violence and aphasia. For the purposes of this scoping paper, we have taken a narrower, and standard definition of mental illness: disorders that affect cognition, mood and behaviors, including depression, anxiety disorders, eating disorders and addictive behaviors.

The area of affective computing and its literature on emotion detection from texts was also useful, particularly research (e.g. Calvo and D'Mello 2010; Strapparava and Mihalcea 2014) discussed in Section 4. These literature reviews focus on the computational aspects and do not generally consider the differences between data sources or the interventions. Furthermore, although we found several reviews of eHealth interventions, we have limited this discussion to include only those with NLP features, such as the one by Barak and Grohol (2011) in Section 5.

\subsection{Overview}

Table 1 provides an overview of papers that have mined textual data for insights into the emotional state and mental health of the author. The first column describes the objective of each investigation, and is split by whether they relate to individual texts (e.g. a blog post or tweet), to individual authors (by aggregating the their posts and any other profile information) or overall populations (i.e. to measure broad trends over large communities of authors). The table also describes the source of data used (typically social media, but there are exceptions). Most of the features considered by researchers and algorithms are based on linguistic analysis of text, but this is often supplemented by behavioral data (e.g. login times), social data (e.g. friends and followers) and demographics (e.g. age, gender, location). There are many 
Table 1. Overview of papers that mine text for insight into author's moods and mental health

\begin{tabular}{|c|c|c|c|c|c|}
\hline Objective & References & Data source & Features & Gold standard & Section \\
\hline \multicolumn{6}{|l|}{ Text level } \\
\hline \multirow[t]{2}{*}{ To detect specific emotions } & $\begin{array}{l}\text { Strapparava and Mihalcea } \\
\text { (2008) }\end{array}$ & LiveJournal & Linguistic & Directly self-reported & 3.3 \\
\hline & $\begin{array}{l}\text { Pestian et al. (2012) and task } \\
\text { participants }\end{array}$ & Suicide notes & Linguistic & Manual annotation & 3.5 \\
\hline $\begin{array}{l}\text { To detect mental health } \\
\text { topics }\end{array}$ & Nguyen et al. (2014) & LiveJournal & Linguistic & Indirectly self-reported & 3.3 \\
\hline \multirow{2}{*}{$\begin{array}{l}\text { To detect distress/suicide } \\
\text { ideation }\end{array}$} & Homan et al. (2014) & Twitter & Linguistic & Manual annotation & 3.1 \\
\hline & O’Dea et al. (2015) & Twitter & Linguistic & Manual annotation & 3.1 \\
\hline To measure stigma & Li et al. (2015) & Weibo & None (observational) & Manual annotation & 3.4 \\
\hline To triage concerning content & $\begin{array}{l}\text { Milne et al. (2016) and task } \\
\text { participants }\end{array}$ & ReachOut & Linguistic, behavioral & Manual annotation & 3.6 \\
\hline \multicolumn{6}{|l|}{ Author level } \\
\hline To measure mood valence & Sadilek et al. (2013) & Twitter & $\begin{array}{l}\text { Linguistic, behavioral, } \\
\text { social }\end{array}$ & None & 3.1 \\
\hline $\begin{array}{l}\text { To measure emotion } \\
\text { contagion }\end{array}$ & $\begin{array}{l}\text { Coviello et al. (2014), Kramer, } \\
\text { Guillory and Hancock } \\
(2014)\end{array}$ & Facebook & Linguistic & None & 3.2 \\
\hline \multirow[t]{2}{*}{ To detect depression } & De Choudhury et al. (2013) & Twitter & $\begin{array}{l}\text { Linguistic, behavioral, } \\
\text { social }\end{array}$ & Directly self-reported & 3.1 \\
\hline & $\begin{array}{l}\text { Coppersmith et al. (2015) and } \\
\text { participants }\end{array}$ & Twitter & Linguistic & Directly self-reported & 3.1 \\
\hline
\end{tabular}


Table 1. Continued

\begin{tabular}{|c|c|c|c|c|c|}
\hline Objective & References & Data source & Features & Gold standard & Section \\
\hline \multirow[t]{2}{*}{$\begin{array}{l}\text { To predict post-partum } \\
\text { depression }\end{array}$} & $\begin{array}{l}\text { De Choudhury, Counts and } \\
\text { Horvitz (2013a) }\end{array}$ & Twitter & $\begin{array}{l}\text { Linguistic, behavioral, } \\
\text { social }\end{array}$ & Indirectly self-reported & 3.1 \\
\hline & $\begin{array}{l}\text { De Choudhury and Counts } \\
\text { (2014) }\end{array}$ & Facebook & $\begin{array}{l}\text { Linguistic, behavioral, } \\
\text { social }\end{array}$ & Directly self-reported & 3.2 \\
\hline \multirow{2}{*}{$\begin{array}{l}\text { To detect distress/suicide } \\
\text { ideation }\end{array}$} & Homan et al. (2014a) & TrevorSpace & Social & Directly self-reported & 3.4 \\
\hline & $\begin{array}{l}\text { Masuda, Kurahashi and Onari } \\
\text { (2013) }\end{array}$ & Mixi & $\begin{array}{l}\text { Demographic, behavioral, } \\
\text { social }\end{array}$ & Indirectly self-reported & 3.4 \\
\hline \multicolumn{6}{|l|}{ Population level } \\
\hline \multirow[t]{5}{*}{ To measure mood valence } & Golder and Macy (2011) & Twitter & Linguistic & None & 3.1 \\
\hline & Kramer $(2010)$ & Facebook & Linguistic & External statistics & 3.2 \\
\hline & Dodds et al. (2011) & Twitter & Linguistic & None & 3.1 \\
\hline & Mitchell et al. (2013) & Twitter & Linguistic & External statistics & 3.1 \\
\hline & Schwartz et al. (2013) & Twitter & Linguistic & External statistics & 3.1 \\
\hline To detect specific emotions & Larsen et al. (2015) & Twitter & Linguistic & External statistics & 3.1 \\
\hline To measure depression & $\begin{array}{l}\text { De Choudhury, Counts and } \\
\text { Horvitz (2013b) }\end{array}$ & Twitter & $\begin{array}{l}\text { Linguistic, behavioral, } \\
\text { social }\end{array}$ & External statistics & 3.1 \\
\hline
\end{tabular}


different approaches for obtaining ground truth data to supervise and evaluate; it can be obtained directly from text authors by explicitly asking them to self-diagnose (e.g. by completing a survey), or indirectly from their behavior (e.g. by joining a depression support group). Some researchers rely on arduous manual annotation, whereas others do not use ground truth at all, and instead perform observational studies.

At the text level, Pestian et al. (2012) host a shared task for mining emotions from suicide notes. Nguyen et al. (2014) separate out LiveJournal posts that discuss depression and related topics. Homan et al. (2014) and O'Dea et al. (2015) detect posts containing suicide ideation and distress, and Li et al. (2015) investigate unhelpful, stigmatizing reactions to suicide on the Chinese social media platform Weibo. Milne et al. (2016) host a shared task for identifying and prioritizing concerning content on ReachOut.com's peer support forum.

At the author level, Sadilek et al. (2013) measure temporal changes in mood valence of Twitter users, whereas Coviello et al. (2014) and Kramer et al. (2014) investigate how moods spread across social connections in Facebook. The various works of De Choudhury et al., and the participants of the shared task hosted by Coppersmith et al. (2015) all attempt to make clinical diagnoses (for depression, posttraumatic stress and postpartum depression) from social media data. Homan et al. (2014b) and Masuda et al. (2013) aim to identify people who are in current distress or are contemplating suicide.

Most of the population-level studies use rough sentiment analysis to measure the mood valence (i.e. positive or negative affect) of Twitter and Facebook, and analyze text features almost exclusively. Golder and Macy (2011) and Dodds et al. (2011) are purely observational, but the others check that geographic and temporal variations correlate well against external statistics. For example, Schwartz et al. (2013) and Larsen et al. (2015) compare against surveys of life satisfaction, De Choudhury et al. (2013b) use depression incidence and prescription rates for antidepressants, and Mitchell et al. (2013) compare against gun violence, wealth, obesity and several other indexes.

Table 1 also lists the gold standard for each study. The gold standard is the dataset used to compare against. In some cases, it is directly self-reported by the text author (e.g. via a mood diary) or indirectly self-reported via their behavior (e.g. joining support group for anxiety). In other cases, the researchers must perform manual annotation, or cross-reference the texts with external statistics such as prescription rates of antidepressants.

\section{Data}

This section describes the data sources that researchers have used for NLP, focusing on texts from which one might infer the author's mood, or diagnose a mental health issue. Considering that Tweets, blog and social media posts differ in many quantitative facts: length of texts, vocabulary used and other features that must be considered for processing, this section aims to distinguish the types of media used. Furthermore, different data sources also differ in their reason people write them. 
We start with research on datasets not explicitly focused on mental health; studies that look for emotions and for subtle cues of mental health in general day-to-day sources such as Twitter (Section 3.1), Facebook (Section 3.2), blogs (Section 3.3) and other social media (Section 3.4).

In Section 3.5, we discuss a series of studies that used suicide notes as the data source and describe how they were collected and used to train algorithms that, for example, detect real from fake ones. Other studies, such as those described in Section 3.6 have used texts written by people with mental illness seeking help and recovery, for example, personal diaries and social sharing of their problems. The benefits of writing for mental health has been recognized in the literature Chung and Pennebaker (2007), Pennebaker and Chung (2007), Pennebaker, Kiecolt-Glaser and Glaser (1988) and many people struggling with depression or anxiety write about their thoughts and experiences. Some choose to do so in online support groups and forums (described Section 3.6), making them available for researchers to analyze.

\subsection{Twitter}

Twitter - one of the most popular social networking or 'micro-blogging' sites distinguishes itself as a source of textual data by accessibility and sheer volume. Almost all activity on Twitter is public by default, and its users simply broadcast their messages to whoever wants to listen. This openness yields a huge source of data - some ninety million tweets per day - that researchers have been quick to capitalize on.

Each tweet is a short 140 character message posted by an individual. Twitter's Application Programming Interfaces (APIs) allow for real-time monitoring, so it is possible to track patterns over time with very low latency. Only a fraction of tweets $(<1 \%)$ contain geo-location data, but it is possible to (roughly) locate tweets using information from the poster's profile. The patterns of following, replying to and otherwise engaging with accounts forms a social network of sorts that can be mined in addition to Twitter's textual, temporal and geographical features.

Most studies were limited to Twitter's free APIs, which deliver either a $1 \%$ random sample (known as the garden hose) or require specific vocabulary of terms and accounts to be monitored. Twitter's terms and conditions prohibit redistribution, so there are no shared datasets and very little replication or direct comparison between the studies described below. While it is possible for researchers to share Twitter data without violating these conditions, there remains the question of whether it would be ethical to do so because of difficulties in anonymizing data Horvitz and Mulligan (2015).

Many studies of Twitter, such as Signorini, Segre and Polgreen (2011) and Paul and Dredze (2011) focus on physical health and disease, and are thus beyond the scope of the current discussion. Also, out of scope are the many other studies, which investigate feelings and emotions as they pertain to a specific subject (e.g. a product or a politician). This section focuses on investigations that address the mental health and emotional wellbeing of the people who tweet. 
One might expect that tweets would provide limited insight given their brevity and public character. It is perhaps surprising then, that many researchers have successfully utilized Twitter data as a source of insights into the epidemiology of emotions (e.g how they propagate) and mental illness. For example, Golder and Macy (2011) used 509 million publicly available posts written by 2.4 million users from across the globe over a period of about two years. They showed that moods are more positive in the morning and decay during the day. They also found that people are happier on weekends and that seasonal moods change with day-length. These results hold few surprises we all know how much we love our weekends and crave sunlight but they do start to validate Twitter as a reliable signal of affective functioning.

Schwartz et al. (2013) also validated the use of Twitter data in characterizing geographic variations in wellbeing, compared to traditional phone surveys about life satisfaction (Lawless and Lucas 2011). In this experiment, about a billion tweets were gathered and, where possible, mapped onto counties in the United States. The paper does not describe exactly how many tweets were successfully geo-located, but the resulting dataset included 1,300 counties for which they found at least thirty twitter users who had each posted at least 1,000 words. The authors then compared the Twitter data against phone interviews and demographics data for the area (socioeconomic surveys and Census). Topic models created with the Twitter data improved the accuracy of life satisfaction predictions based on the demographic controls (county-by-county scores for age, sex, ethnicity, income, education) which were in turn more predictive than the prevalence of words from emotion lexicons. In combination, the three approaches achieved a Pearson correlation of 0.54 with the scores obtained through random direct surveys of people in a county, while the controls on their own achieve only 0.44 correlation. We Feel (Larsen et al. 2015) is a system that analyzes global and regional changes in emotional expression. The evaluation consisted of $2.73 \times 109$ emotional tweets collected over twelve-weeks, and automatically annotated for emotion (using Linguistic Inquiry and Word Count (LIWC)), geographic location and gender. The analysis confirmed regularities found in emotional expressions in diurnal and weekly cycles and these were reflected in the results for a PCA: The first component explained $87 \%$ of the variance and provided clear opposite loadings between positive and negative emotions. This study adds to the evidence that Twitter and possibly other publicly available social media data can provide insights into emotional wellbeing and illness across different populations.

De Choudhury et al. (2013b) built a classifier for determining whether a Twitter post indicates depression. For the purposes of the current discussion, it is sufficient to say that the classifier produced a score that is higher for tweets that indicate depression than those that did not. The paper also describes a Social Media Depression Index (SMDI), which scores a group of tweets (e.g. those by a single author, or those from a city) by the prevalence of high-scoring tweets. One experiment in this paper is similar to the Schwartz et al. (2013) study described above, but in this case the tweets were geo-located down to state level rather than county. The SMDI score was calculated for each state, and these scores achieved a 0.51 correlation against ground truth (defined as the data used as the training set) based on the Centre for Disease Control (CDC) calculations. It is important to note that this 
score is obtained entirely through analysis of Twitter; unlike (Schwartz et al. 2013) it does not make any use of background demographic data. In the same paper, the authors also calculated the SMDI scores for the twenty "unhappiest US cities" and achieved a strong positive correlation (0.64) with the prescription rates for common antidepressants.

Many other studies follow a similar pattern of quantifying topics and emotions over entire populations using Twitter. For example, Dodds et al. (2011) introduced the Hedonometer, which cross-referenced a truly massive dataset of 4.6 billion tweets against their "Language assessment by Mechanical Turk" (LabMT) vocabulary of happy and sad terms. Like Golder and Macy (2011), their analysis of the resulting signal reveals unsurprising but reassuring patterns, such as increased happiness during the weekend. Mitchell et al. (2013) applied the Hedonometer using only tweets with exact GPS coordinates. At a state level, they found moderate correlations with incidences of gun violence $(r=-0.66)$, the American health index $(r=0.58)$, and the US Peace Index $(r=0.52)$. At a city level, they also found associations amongst Hedonometer levels and indexes of wealth and obesity.

However, the studies described above consider only the broad picture. Analysis at this level may be more forgiving, because mistakes can be averaged out as tweets are aggregated across entire states, counties and cities. For researchers, this poses the question: Are Twitter-derived measurements accurate enough to drill down to an individual?

One group from Microsoft Research has demonstrated two distinct approaches for conducting studies with individual Twitter users. In the first experiment, De Choudhury et al. (2013) developed a classifier that estimates the risk of a Major Depressive Disorder (MDD) before it happens. To gather a gold-standard dataset, they conducted a crowdsourcing task that required 1,583 volunteers to complete diagnostic surveys - specifically the Center for Epidemiologic Studies Depression Scale (CES-D) and the Beck Depression Inventory (BDI) - and answer questions about their basic demographics and history with depression. The same task invited (but did not require) participants to provide details of their twitter accounts. The task yielded two datasets; 305 twitter accounts with no indication of depression, and 171 accounts that scored highly on the CES-D survey and had also been diagnosed with depression at least twice in the last year (a requirement to qualify for MDD). A total of 2.1 million tweets (an average of 4,533 tweets for each account) were captured within the year prior to the crowdsourcing task. From this data, they identified behavioral features about engagement, emotion, language styles and medication used. These features were used to train the classifier, and distinguish between the depressed and non-depressed accounts. To summarize their findings, the depressed user accounts were

- less likely to tweet or respond to others' tweets,

- more likely to tweet late at night,

- more likely to use first-person pronouns (i.e. tweet about themselves),

- less likely to use third-person pronouns (i.e. tweet about others),

- less likely to follow others or gather followers. 
In addition to these social and behavioral differences, the study also identified a lexicon of terms that were more common among the depressed accounts, such as mentions of medications, depressive symptoms and words related to disclosure. All of these signals were combined with basic demographic features (age, gender, income and education level) to develop a classifier that could distinguish between depressed and non-depressed accounts with a recall of $63 \%$ (the fraction of accounts that are automatically detected) and precision of $74 \%$ (the fraction that is labeled correctly).

In another study, the same authors focused on measuring behavioral and emotional changes that could be indicative of postpartum depression (De Choudhury et al. 2013a). Rather than surveying new mothers directly, they instead monitored Twitter automatically for birth announcements, using newspapers as a model. This identified 376 mothers after manual verification via crowdsourcing. The tweets of each mother were gathered for the three months prior to the birth notice and another three months after. In all, the dataset contained $37 \mathrm{k}$ prepartum and $40 \mathrm{k}$ postpartum tweets. These were analyzed as described above (De Choudhury et al. 2013). Each mother was characterized with 33 features, and the ground truth (the training set) was based on a heuristic threshold of changes, rather than a direct questionnaire of depression. Instead the analysis identified mothers for whom twitter-derived measures of social engagement, linguistic style, etc. - the same signals that indicated depression in the previous study - changed dramatically between the pre and postpartum periods. It was assumed that these were mothers who were not adjusting well to parenthood, and were displaying signs of postpartum depression. A classifier was built that could from the prepartum data alone predict the mothers who would later exhibit these dramatic changes with an accuracy of $71 \%$.

Coppersmith et al. (2015) host a shared task that also attempts to make clinical diagnoses from Twitter data; in this case, for depression and posttraumatic stress disorder. Their work, and that of the task participants, is described in Section 4.4.

Twitter posts can be combined with geo-location information to produce approximate models of people's emotion and context. For example, Sadilek et al. (2013) used information from 6,237 users who shared their GPS location. They used linguistic features (i.e. based on the Linguistic Inquiry and Word Count LIWC tool), behavioral (such as the number and time of tweets), social network (i.e. friends) and a summary quantity calculated from LIWC that categorized users into three states: positive, neutral and negative. By using this data, they found evidence of temporal mood patterns, demonstrated emotional contagion in groups and were able to predict when users were going to be in one of those three states (positive, neutral, negative) over the next ten days. Another study using the same dataset (Homan et al. 2014) selected 2,000 tweets around suicide risk factors (1,370 from the LIWC sad dimension and 630 with suicide specific terms) and annotated them. The annotations were performed by a novice and counseling psychologists and then judged by another novice. Each of the tweets was annotated as happy, no distress, low distress and high distress. The authors then trained a Support Vector Machine (SVM) to classify the tweets into the four categories and found F1-measures of 0.4-0.6 (the harmonic mean of precision and recall). 
O’Dea et al. (2015) aim to separate out genuinely concerning tweets mentioning suicidal ideation from the large amount of flippancy and hyperbole that exists on the platform. They gathered a corpus of 2,000 tweets containing phrases like kill myself and tired of living and manually coded them as strongly concerning (14\%), possibly concerning $(56 \%)$ and safe to ignore $(29 \%)$. Agreement between annotators ranged between 0.47 and 0.64 kappa, indicating that the task was strongly subjective. An automated SVM classifier was able to automatically separate strongly concerning tweets with a precision of $80 \%$ but a recall of only $53 \%$.

\subsection{Facebook}

Facebook is another huge potential source of data. It is used by an estimated 1.13 billion people each day, ${ }^{1}$ and has become a major platform for promoting mental health campaigns and recruiting research participants to mental health studies. This section focuses on attempts to measure the emotional state of Facebook's users.

Although Twitter users simply broadcast their messages to anyone who will listen, Facebook users exert more control to form closed audiences of friends and family members. This increased privacy may increase the user's openness and honesty. Therefore, while Facebook data may be better suited to monitoring emotional state, it is more difficult to obtain by researchers outside Facebook.

Kramer (2010) analyzed 400 million status updates from English speaking users within the United States, and developed a measure of gross national happiness by scoring each status update against the positive and negative terms within it (as given by the Affective Norm for English Words (ANEW) vocabulary described in Section 4.2). The measure was roughly validated in a similar fashion as Golder and Macy (2011), by visually inspecting the data for expected patterns like peaks during holiday periods and weekends and troughs during national disasters. A more rigorous validation was conducted by recruiting 1,300 Facebook users to complete a survey about life satisfaction. However, the happiness scores derived from their status updates achieved only a weak correlation with life satisfaction survey scores $(\mathrm{r}=0.17)$. It is not clear whether this weak association was due to the simplicity of the algorithm (understandable, given the scale at which it was run), or to insufficient data (some of the Facebook users involved had as few as three status updates) or to factors beyond the scope of the study design.

Also relevant to this discussion is the study completed by Coviello et al. (2014) which explored how negative emotion spreads as a contagion between Facebook accounts. The experiment investigated rain as a trigger for changes in valence, which was measured by the prevalence of known positive and negative terms within status updates (similar to Kramer 2010). The experiment demonstrated that not only do people post fewer positive and more negative posts during rainy days, but also that these posts have a statistically significant effect on the valence of their friend's posts. Positive posts promoted positive posts and inhibited negative ones, and vice-versa; even when these friends were not experiencing the same weather patterns.

${ }^{1}$ Updated statistics are available at http://newsroom.fb.com/company-info 
Another study of emotional contagion in Facebook showed how small changes in the filtering algorithm used in the Facebook Newsfeed can have a significant impact on the moods of users (Kramer et al. 2014). The experiment compared two filtering algorithms; one reducing the number of positive status updates a user was exposed to, and another suppressing negative updates. The valence of a post was again identified using a similar approach as in Kramer (2010). In total, $690 \mathrm{k}$ users were randomly selected and exposed to these algorithms. Approximately, $155 \mathrm{k}$ participants within each condition posted at least one status update within the week. From these status updates, the authors measured a small $(<0.1 \%)$ but statistically significant shift toward positive terms (and away from negative ones) among those exposed to fewer negative posts. The opposite held true for the positivity-reduced condition. The study contributed interesting insights into how emotions propagate on social networks and the impact of design on people's emotions, two underserved areas of research. But the study triggered an understandable flurry of outrage and concern for failing to inform or obtain consent from participants before attempting to manipulate their moods, as reflected in their Facebook updates (Calvo, Peters and D'Mello 2015).

Moreno and Jelenchick (2011) manually evaluated a year's worth of status updates of 200 college students. Each update was coded against the DSM IV criteria for depression. The authors reported that $25 \%$ of profiles contained at least one depressive post, and five profiles had periods of multiple depressive posts each spanning several months; the authors considered these periods to match DSM criteria for a major depressive episode. This figure was expected, given $30 \%$ of college students report feeling depressed and unable to function each year (American College Health Association 2009).

The underrepresentation of depression terminology in the Facebook posts would seem to indicate that few people are comfortable sharing their depressive symptoms explicitly on Facebook. This does not invalidate Facebook data as a means of recruiting information that could be developed into a diagnostic tool, but it does point out the need to look for subtler cues. To our knowledge, the only published attempt to do so automatically is by De Choudhury and Counts (2014), who recruited new mothers willing to share their Facebook data. In total, they gathered twenty-eight mothers who had been diagnosed with postnatal/postpartum depression (PPD) and 137 mothers with no experience of depression (a PHQ-9 Patient Health Questionnaire, was used to exclude mothers who displayed symptoms of depression but had not been clinically diagnosed with PPD). Their accounts were mined for activity for fifty weeks prior to and ten weeks after birth.

From this data, the authors observed that mothers suffering from PPD were

- less likely to make posts or share media,

- less likely to engage with or tag media left by others,

- less likely to receive comments or likes from friends,

- more irregular in the times that they interacted,

- more likely to use first person pronouns (i.e. talked more about themselves),

- more likely to ask questions. 
Interestingly, the researchers did not find a statistically significant difference in the use of positive or negative emotion terms, as measured by LIWC. This is inconsistent with their previous work with Twitter (De Choudhury et al. 2013a), one explanation would suggest that Facebook posts are less emotionally expressive than tweets. Unfortunately, we cannot make any direct comparisons between Twitter and Facebook, because the authors did not develop or evaluate a binary classifier in the same way.

\subsection{Blogs and journals}

Blogs are used by many as personal diaries, and as a way to reflect and to share daily experiences. Increasingly, people can annotate their own posts with metadata (i.e. labels) about their emotions. Livejournal.com was one of the first to provide this self-annotation feature to users and it has been used in multiple studies. For example, Strapparava and Mihalcea (2007) extracted a corpus of 8,671 LiveJournal posts labeled (by the post authors) with six emotions. The study sits slightly outside of the scope of this review, however, because they did not attempt to classify the blog posts themselves or the emotional state of their authors. Instead the posts were used as background training data to classify news headlines (from the SemEval 2007 dataset) and the emotion they were intended to provoke in the reader. Nevertheless, this work is described in more detail in Section 4.4.

Nguyen et al. (2014) aim to automatically separate out LiveJournal posts that talk about tough times. For ground truth, they extracted a depression dataset of $38 \mathrm{k}$ posts from sub-communities for depression, self-harm, bereavement, etc. and a control set of $230 \mathrm{k}$ posts from other, less dire sub-communities. They experimented with a wide range of features, including LIWC (linguistic, social, affective, cognitive, perceptual, biological, relativity, personal concerns and spoken), affect (also based on LIWC) and topic models generated by LDA. They were able to automatically determine whether each individual blog post belonged to the depression or control set with an accuracy of $93 \%$, and the best features were the LDA topics.

\subsection{Other social media data}

As new social media platforms are created they are used in mental health studies. For example, the nature of personal disclosure has been studied on Reddit (De Choudhury and De 2014), an online media platform similar to discussion forums but with an optional "throwaway" account type that improves anonymity. The results suggest that anonymity promotes disclosure, despite the often caustic nature of the discussions generated around a post. Although the number of throwaway accounts is proportionally small, the fact that $61 \%$ were used only once suggest these users do not want to leave any trail behind, but as the authors point out, this also means they cannot receive much social support.

Different countries often have different languages. They also vary on their socioeconomic variables, their conceptions of mental health, stigma and therefore support of those who are ill. Therefore, studying the social networks popular in 
different countries is important. Mixi, the most popular social network platform in Japan, which is the Organization for Economic Co-operation and Development (OECD) country with the highest suicide rate, was used to study the relationship between a person's social network (i.e. relationships) and suicide ideation (Masuda et al. 2013). According to the authors the effect of the age, gender and number of friends on suicide ideation was small.

Another interesting perspective is to look into peoples' attitudes toward suicide (e.g stigma) as they can inform the type of suicide prevention interventions. A study using data from Weibo, a Chinese social network platform (Li et al. 2015) analyzed the social attitudes of people publicly commenting on others who made public their intention to commit suicide and found stigma was widespread and terms such as deceitful, pathetic and stupid were often used.

Those who suffer stigma maybe at risk of mental health issues and their communities might require special attention, for example, researchers have studied TrevorSpace, a social network popular amongst lesbian, gay, transgender and bisexual communities (Homan et al. 2014a). The study did not use the text but only the connections amongst individuals and showed that some features are predictive of distress or mental-ill health.

\subsection{Suicide notes}

Suicide notes were first studied in the nineteenth century by Durkheim, a pioneer of suicide risk research (Durkheim 1897). Shneidman created the field of suicidology around 1949 and collected and systematically studied suicide notes (Shneidman and Farberow 1957). In 1957, Shneidman and Farberow published a book that explored the reasons why people kill themselves, and collected genuine suicide notes paired with fake suicide notes written by a healthy control group that were demographically matched to the suicidal authors. The aim was to explore the difference between why people think others suicide, and why individuals actually do.

Pestian et al. (2010) extracted from this book a corpus of thirty-three genuine (written by people who completed suicide) and thirty-three fake notes (written by people simulating a suicide note). They showed how difficult they are to distinguish manually: In their experiments, mental health professionals achieved an accuracy of $63 \%$, whereas psychiatry trainees were only $49 \%$ accurate (i.e. worse than random chance). The authors were able to train a classifier that achieved $74 \%$ accuracy. However, the classifier relied somewhat on emotion annotations (e.g. guilt, hopeless, regret) that were made by a panel of three mental health professionals. The authors did not report the accuracy of the algorithm without these handcrafted annotations.

Removing this reliance on expert annotation appears to be the focus of Pestian et al. (2012), which presents a shared task for annotating suicide notes. Shared tasks are common computer science activities where research groups compete to solve the same problem. The task used a corpus of genuine notes written by people who took their own life, collected between 1950 and 2011. The notes were manually transcribed, anonymized and carefully reviewed, before being shared with a panel of 1,500 "vested volunteers" who were recruited from online communities 
and Facebook pages created to support grieving friends and family members. These volunteers were asked to annotate the notes for occurrences of negative sentiments (abuse, anger, blame, fear, guilt, hopelessness, sorrow), positive sentiments (forgiveness, happiness, peacefulness, hopefulness, love, pride, thankfulness) and neutral sentiments (instructions, information). In total, 900 suicide notes were each annotated separately by three volunteers.

In this shared task, 106 researchers in twenty-four teams tried to automatically reconstruct these manual annotations. They were given a training set of 600 annotated letters to develop their algorithm, which was evaluated on the remaining 300 letters (these were not released until algorithms were finalized).

There are two ways of calculating Precision, Recall and their harmonic mean F1 (a commonly used measure of classification accuracy). Macroaverages of these are the simple average over classes. Microaverages are obtained pooling per-document (i.e. post) decisions across classes, and computing $\mathrm{P}, \mathrm{R}$ or F1 on the pooled contingency table. All of the top 10 competing teams achieved microaveraged F1-measures between $53 \%$ and $62 \%$. An ensemble combining several of the systems could theoretically achieve an F1-measure of $76 \%$. Section 4.4 describes the approaches of the top few teams. The F1-measure has its best value at 1 and worst score at 0 . Microaveraged F1 was used to rank the teams.

This shared task used suicide notes written offline, mostly before the Internet era. But research on suicide notes written for the Internet have shown consistent findings with those written offline (Barak and Miron 2005) and has been used as evidence for the development of support services (Barak 2007).

Writing about suicide in the Internet age brings new challenges. Christensen et al. (2014) conducted a review of research studies that focused on three particular challenges: the use of online screening for suicide, the effectiveness of eHealth interventions aimed to manage suicidal thoughts and newer studies aimed to proactively intervene when individuals at risk of suicide are identified by their social media postings. The review highlighted the need for more evidence on the effectiveness of eHealth interventions for suicide prevention.

\subsection{Online forums and support groups}

Online peer-to-peer communities and support groups are one of the most promising ways of reaching out to more people, and allow them to discuss their health issues (Eysenbach et al. 2004). These forums may be particularly good for reaching young people (O'Dea and Campbell 2010). Although the evidence on their efficacy is not yet strong, this is due in part to the difficulty of running systematic trials (Eysenbach et al. 2004; Pistrang, Barker and Humphreys 2008). One of the limitations of peer support style groups is that they need trained moderators who can help manage the community and can provide informed feedback when needed. When the communities grow this becomes a challenge.

The CLPsych 2016 shared task (Milne et al. 2016) aimed to address such challenges of scale by allowing moderators to focus their efforts where it is most needed. It collected forum posts from ReachOut.com - a site for young Australians facing 
tough times - and asked participants to automatically triage them as green (no intervention required), amber (a moderator should ideally respond, but not urgently), red (a moderator should respond as soon as they can) or crisis (the post indicates someone is at risk of harm).

The task attracted sixty submissions from fifteen teams of researchers. Each team was initially given 947 annotated posts to develop and train their algorithms, and later given 280 posts - with annotations only seen by the task coordinators - for evaluation. The three best performing teams obtained a macroaveraged $\mathrm{f}$ measure of 0.42 , but used very different approaches to do so. Kim et al. (2016) combined relatively few features (unigrams and post embeddings) with an ensemble of SGD classifiers. Brew (2016) used traditional n-gram features with a well-tuned SVM classifier, and achieved the best separation of urgent posts (crisis and red versus amber and green) with 0.69 f-measure. Malmasi et al. (2016) gathered a larger number of features from not only the post itself, but also the preceding and following ones, and achieved the best separation of flagged posts (crisis, red and amber versus green) with $0.87 \mathrm{f}$-measure. The dataset is available ${ }^{2}$ for researchers to continue developing their algorithms.

This triage task was a continuation of Moderator Assistant (Liu et al. 2013) a system that uses NLP to detect people in distress and helps moderators prioritize their workload (described in more detail in Section 5.5).

Other papers have analyzed patients' self-narratives and provide further evidence of the potential of NLP techniques in PTSD diagnosis. For example, He, Veldkamp, Glas, and de Vries (2015) collected in an online survey in a forum for those seeking mental health aid and evaluated methods using n-grams and unigrams with Decision Trees, Naiïve Bayes, SVM and Product Score Model (PSM). Unigrams with PSM had the highest accuracy (0.82).

\subsection{Summary}

- Most popular forms of social media have been used as data sources for mental health applications.

- Number of users, language (i.e. English) and availability of APIs increase the chances of a platform being used. Twitter is the most widely used source of data mainly because the collection of public data is easy. Facebook is also common, often used by authors who also work for (or in partnership with) the company.

- We only searched papers in English, and these mostly talked about content written in English. A few exceptions (e.g. Japanese) are mentioned. It would seem that NLP in non-English languages is an unexplored area. This may be related to the lower quality, or absence of NLP tools in languages other than English.

${ }^{2}$ Researchers can apply for access to the ReachOut triage dataset at http://bit.ly/triage-dataset 
- One of the most studied corpora of user-generated texts is a suicide notes collection because it was used in a shared task competition. Share tasks allow researchers from multiple disciplines to collaborate on a particular problem.

\section{Automated labeling}

We use the term labeling in this section to mean the identification of emotions, moods and risk profiles that might indicate mental health problems or profiles that could be used to target interventions. It goes beyond diagnosis, as the term to refer to DSM -V classifications of mental illness, although we do note when studies attempt to achieve specific psychiatric diagnosis. Labeling is generally done using document classification techniques that take certain features as input and map them to a set of labels. The techniques used include tokenization, feature extraction and selection, parsing and machine learning classification. We provide a summary of key publications organized according to the tasks required to build an automated labeling system.

\subsection{Gathering training data}

Often the first step to build a classifier that can automatically detect emotions and mental health issues is to gather labeled data. This is a requirement for supervised machine learning algorithms that use this data for training and evaluation. The challenges of collecting and labeling data, particularly in realistic scenarios (i.e. "in the wild") include time and cost (proportional to the amount of data being labeled), validity (particularly dependent on how the data was collected) and reliability (often requiring multiple trained annotators per document). These challenges have been discussed elsewhere, for example, in the literature on Affective Computing (Riva, Calvo and Lisetti 2014). Semi-labeled and unlabeled data can also be used to train classifiers. For example, using Expectation-Maximization and Naive-Bayes classifiers (Nigam et al. 2000) contributed a technique that reduced classification errors by up to $30 \%$.

\subsection{Feature extraction}

The literature would suggest that a combination of language and other forms of features is the most promising. This will likely be dependent on the application. This section describes these in more detail

Demographic features. Textual data can be complemented with socio-demographic data of individuals. Variables like county-by-county scores for age, sex, ethnicity, income, education and geo-location have been used in studies showing that these features can improve the accuracy of the classifier (Schwartz et al. 2013). These results are in line with evidence suggesting that social expressions of emotion are age and gender dependent, while location influences time and weather, which in turn, can also influence emotions.

Lexical features. Emotions are often inferred by the percentage of emotional terms in the posts. These are often computed using LIWC (Pennebaker et al. 2015; Tausczik 
and Pennebaker. 2010). LIWC is a software tool that provides psychologicallygrounded lists of positive and negative emotional terms (amongst other categories reflecting writing style). The assumption, which is supported by research evidence (Strapparava and Mihalcea 2014; Pennebaker 2011), is that emotion terms reflect emotion feelings (i.e. if you use more positive words, it is because you feel more positive). Language and feelings are indeed related, however, it appears that the relationship is weak. LIWC counts the number of words in a category (e.g. affective or social). The words are added to a category by the developers who often provide 'validity judgments' the correlations of judges ratings of the category with the LIWC variable. In particular, the correlation between the specific emotional words in LIWC and the emotional ratings by human 'validity judges' is a modest 0.41 for positive and 0.31 for negative terms; and these are amongst the lowest in the LIWC dictionaries (Pennebaker et al. 2015).

Emotion features from LIWC are often taken as good approximations of what people are feeling, thus, LIWC has been used in several mental health projects. But since the labeling is not done for clinical purposes the thesaurus does not directly indicate if, for example, a user wrote the word sad, has a higher probability of illness as would be measured by PHQ9 score or by the diagnosis of a clinician. Labeling of this type is generally done for a subset of each corpora or other information for the ground truth. For example, Kramer et al. (2014)'s study that manipulated Facebook News Feed filters (described in Section 3.2) used emotion terms from LIWC. Other emotional thesauri include WordNet (Miller et al. 1990), particularly its extension WordNet-affect (Strapparava and Valitutti 2004) and normative databases such as the Affective Norm for English Words (ANEW) (Bradley and Lang 1999). These thesauri can be used to group words, emotion words, for example, according to their valence or activation (i.e. arousal) (Calvo and Kim 2013).

Behavioral features. When people write they leave a trace of their behaviors. This metalinguistic information includes the time they wrote, for how long, if the writing was reply to another post, etc. The study by Sadilek et al. (2013) using geo-location and twitter data (discussed in Section 3.1) is an example of how this type of data can be used in the modeling of moods.

Social features. These include the number of relationships (e.g. Facebook friends) and the topological map (i.e. who is related with who) that have been found to correlate to emotional and mental health constructs. The study by Sadilek et al. (2013) used social features to model emotional contagion within groups. Others have shown how they can be used to improve the prediction of mental health states (Masuda et al. 2013; Homan et al. 2014).

\subsection{Feature selection techniques}

Feature selection is required to reduce the number of features (i.e. the input space) used in the classification task. Without feature selection classification algorithms suffer from overgeneralization and are often less efficient (Witten and Frank 2005). There are multiple ways of reducing the dimensionality of the problem including Principal Component Analysis, regression models and other statistical approaches. 
In text classification, Bi-normal separation (BNS) methods outperformed all other methods in an evaluation of twelve feature selection methods (e.g. Information Gain) over a benchmark of 229 text classification problems (Forman 2003). The same study compared different techniques when only one dataset was available (the most frequent scenario) and showed that generally BNS outperformed other approaches. The exception was when precision was the goal in which case Information Gain yielded the best results.

\subsection{Evaluating labeling systems}

There are too many machine learning classification techniques to be discussed here, so we limit our discussion to those that have been used in mental health research. Complete descriptions of the algorithms can be found in machine learning textbooks or reviews (Sebastiani 2002; Strapparava and Mihalcea 2014).

One of the most comprehensive ways to evaluate and compare classification systems through shared task competitions between independent research groups. This approach is used in the computer science and NLP communities to help identify the most accurate system. To make the process fair and reliable, the training data for the system is shared with the competing teams, but the test data is only made available to the shared task teams after the evaluation of the algorithms has been completed.

The suicide notes task described earlier is possibly the best example of shared task competitive challenges applied to mental health data. However, this approach has also been applied to related emotion detection tasks. For example, the SemEval2007 task (Strapparava and Mihalcea 2007), where emotions were detected in a collection of news stories. Albeit not on mental health and not covered here, the SemEval-2007 and other similar shared tasks provide important insights for those working on mental health applications.

Micro and macro averages of precision, recall and F1-measures were calculated for each team. Microaverages weigh equally all data points so more common categories are weighted more heavily. In the suicide notes shared task, a team from the Open University in the United Kingdom had the best results with a microaveraged F1 $=0.61$, the least performing team had $\mathrm{F} 1=0.30$, showing a large variation in the results when different techniques are used. The teams used different techniques including Part Of Speech (POS) taggers, thesaurus like WordNet (Strapparava and Valitutti 2004), emotional lexicons and LIWC. The winning team's submission (Yang et al. 2012) is an example of the complexity of NLP systems. A text processing tool fed the notes to subsystems that identified instances of emotions, both at the token and the sentence levels. There were components to detect negations, emotion cues and terms. It also used numerous machine learning techniques that have shown to be successful in other applications, including Conditional Random Fields (CRF), SVM, Naiive Bayes (NB) and Maximum Entropy (ME). The final output was the emotion labels at the sentence level.

The runner-up (Xu et al. 2012) also used a combination of techniques. A key distinguishing factor in this submission was perhaps the augmentation of the 
dataset with semantically similar blog posts from LiveJournal and the SuicideProject (where bloggers can annotate data with emotions). They also used CRF and SVM algorithms in a binary fashion - one classifier for each label.

Since the other teams (Cherry, Mohammad and De Bruijn 2012; Luyckx et al. 2012; McCart et al. 2012; Spasic et al. 2012) reported details of their algorithms and outcomes, the shared task also provides insights into the techniques that did not produce satisfactory results. For example, Yu et al. (2012) reported low accuracy using Wordnet, character n-grams and word n-grams (a contiguous sequence of $n$ terms). They concluded that character n-grams and dependency pairs provide good features for emotion detection in suicide notes yet word n-grams and POS n-grams were less robust.

Another shared task was a 2015 Computational Linguistics and Clinical Psychology (CLPsych) shared task (Coppersmith et al. 2015). The data used for the task, and a hackathon held at John Hopkins University, consisted of anonymized Tweets written by 1,746 users who stated a diagnosis of depression or posttraumatic stress disorder (PTSD), with demographically-matched community controls. The shared task consisted of three binary classification experiments: (1) depression versus control, (2) PTSD versus control and (3) depression versus PTSD. The shared task had submissions from four research groups: University of Maryland who focused on topic models (Resnik et al. 2015), University of Pennsylvania which used a variety of methods (Preoţiuc-Pietro et al. 2015), University of Minnesota who used a rule based approach (Pedersen 2015) and a submission by the workshop organizers and Microsoft that used character language models. Due to length limitations, we recommend reading those papers for further details.

A rarer evaluation approach involves studying a classification system while it is in use. Section 5.5.1 describes a triage system that uses the classifiers in real life. Their evaluation should in general go beyond the classifier accuracy and explore aspects such as how users perceived the triage system, and how they evaluate the possible usage scenarios, etc.

\subsection{Summary}

- All feature selection and classification algorithms common in the NLP literature have been tried. Overall, we could not ascertain whether a particular technique was superior to other techniques.

- LIWC is the most widely used tool for extracting features from text.

- An understudied area is that of triage systems used in real time, with real users. Most studies are performed offline, where the diagnosis was not used to respond to actual users.

\section{Interventions}

The Internet has provided a medium for low-cost psychotherapeutic interventions that psychologists have been incorporating into their practice for over a decade. 
In this section, we describe examples of how NLP and Generation are used to automatically create interventions.

Barak and Grohol (2011) provided the taxonomy of Internet-based interventions used in this section: "psycho-educational websites", "Interactive self-guided interventions", "online counseling and psychotherapy", "online support groups and blogs" and "other types", predicting the increase in "Virtual reality", "gaming" and "SMS and texting" therapies.

In a comprehensive review (Barak et al. 2008), the authors considered 92 internetbased intervention studies (and included sixty four in the meta-analysis) and reported a mean weighted treatment effect size of 0.53 . We reviewed the 92 papers, however, only one study (Owen et al. 2005) was found to use NLP techniques. Yet, the effect size was similar to effect sizes reported for traditional face-to-face therapies. The potential of further improving these results by using state-of-the-art NLP techniques is momentous.

The lack of studies describing text-based analysis techniques represents an area full of opportunity for the mental health intervention sector. NLP techniques could augment therapist-based mental health interventions. For example, NLP techniques could potentially be used to detect links between behaviors and emotions described by the client, or to help people recognize unhealthy thinking patterns. Since the number of studies is so small we expanded our scope to include studies from the physical health literature to demonstrate the potential of this area of research.

\subsection{Web-based psycho-educational interventions}

Psycho-education refers to the educational interventions where patients and their families can learn about the illness and the techniques and resources available to help them. Most examples of psycho-education interventions are fixed (i.e. not personalized) descriptions of symptoms and treatment written for a general audience. Although these have been excluded from this review, we explore here how they could be made adaptive and personalized using NLP. Exploring new approaches is important since research shows that web-based psycho-education interventions have therapeutic value and show positive outcomes (Ritterband et al. 2003). Internetbased interventions (CBT, psychoeducation and other) have shown to be effective in treating depression and anxiety (Spek et al. 2007; Donker et al. 2009), eating disorders (Neve et al. 2010), smoking and alcohol consumption (Bewick et al. 2008; Myung et al. 2009) among other conditions.

Other application domains can provide ideas for innovative approaches in mental health. For example, in the same way that NLP has been used in educational technologies one could expect them to be used in psycho-education. Regrettably this has not been the case. A search on Google Scholar for "NLP psycho-education", "computational linguistics psycho-education" did not return any positive results. NLP is used in education, for example, in automated assessment tools where users can answer questions and get formative feedback to improve their understanding. In health this could be done to improve their understanding of an illness. The 
content of websites could also be improved by measuring the complexity of the language used, allowing content editors to adapt the language to different age and socioeconomic groups.

A common marketing strategy is to personalize information with automatically generated text, for example, using simple templates to generate mailouts. Evaluations have shown that this type of semi-automatic and fully-automatic texts (i.e. letter, interventions) compare well with those generated by humans on a number of measures such as tone, rhythm and flow, repetition and terminology (Coch 1996). Within health, personalization has been considered critical to patient-centered care and a number of studies have evaluated Natural language Generation (NLG) techniques in the authoring and personalization of webpages containing patient education materials. Unfortunately, the personalization of materials has been tried only in the context of physical health (e.g. surgical procedures, cancer, etc.). In the context of mental health, personalizing information (i.e. interventions) could also be helpful for family members, moderators of peer-support groups, the general public in social media, etc. Automated text generation methods could provide the moderators with information that they can use for quickly customizing and replying. This may even be useful for mental health clinicians, where information about a specific patient can be presented in the form of a report.

There are several possible tools used to build NLG systems including SimpleNLG (Gatt et al. 2009), a simple Java-based generation framework. The design of these tools is generally informed by the work of Reiter and Dale (2000), who defined a general architecture used in most of the applications we reviewed. The architecture has three components connected together into a pipeline: (1) A Document Planer determines the content and structure of a document. (2) A Microplanner decides how to communicate the content and structure chosen by the Document Planer. This involves choosing words and syntactic structures. (3) Surface Realiser maps the abstract representations used by the Microplanner into an actual text.

One of the few examples of mental health applications is PyschoGen (Dockrey 2007), an NLG-based system that changes its output based on the emotional state (set by the user). The experimental system followed the standard document planning $\rightarrow$ microplanning $\rightarrow$ realization pipeline. The goal of the project was not to send the text generated to real users or even generate highly natural output, but rather explore how emotional data could be generated. This type of approach could be suitable for mental health interventions that express empathy and compassion in line with the concept of client-centric health information and resources. Since there is little research on NLG applications to mental health, we describe here some related to physical health.

"Information Therapy (DiMarco et al. 2007) is a system providing preoperative information, that largely consists of personalizing resources that are normally distributed via brochures - each discussing a surgical procedure. The system was designed using a collection of reusable texts, each annotated with linguistic and formatting information, then the NLG tools automatically selected, assembled and revised the reader-appropriate pieces of text. This approach could easily be adapted to psycho-education interventions. 
Similarly tailored information systems used in diabetes, migraine or cancer could be used to help those trying to learn about mental illness (though perhaps not for the patients themselves). Bental and colleagues (Bental, Cawsey and Jones 1999; Bental and Cawsey 2002) have described a variety of tailored patient education systems that may act as examples. For diabetes patients, PIGLIT provided personalized information about the disease, their hospital etc. (Binstead, Cawsey and Jones 1995). Similar work used hypertext pages for Migraine patients (Buchanan et al. 1995), cancer patients (Jones et al. 1999) and to accompany prescriptions (De Carolis et al. 1996) have also been described. Tailored patient information systems have shown to be more effective than non-tailored computer system (or leaflets). For example, a Randomized Control Trial (RCT) of cancer patients (Jones et al. 1999) ( $\mathrm{N}=$ 525 ) with three conditions (paper brochures, personalized and general information hypertext) showed that more patients offered the personalized pages felt that they had learned more (and used it more), that the information was relevant to them, and even shared the information with others. Not all the results of NLG applications have been positive. A clinical trial generating personalized smoking cessation letters (Reiter, Robertson and Osman 2003) after participants $(\mathrm{N}=2,553)$ responded to a questionnaire indicated that those who received personalized information were no more likely to stop smoking than those who received a non-tailored letter.

\subsection{Interactive, self-guided interventions}

While content in tailored information systems is directed one-way - from the system to the user - similar techniques can be used to produce interactive psychoeducation interventions. This includes interactive software (websites, mobile apps, SMS) that offer an individualized step-by-step structure of information and guidance - a form of online self-help activity. The interactive system may provide a form of education as in the previous category, but may also only provide a structured set of activities for the user to complete. Back in the 1960s, a system called Eliza (Weizenbaum 1966) was one of the first AI systems for this type of intervention. It emulated a Rogerian psychological perspective and used an early form of AI to engage the user in a dialogue of question and answer and asked empathic questions, based on the previous dialogue move. There has been some research around this model, where a software agent plays the role of the therapist (Bohannon 2015).

Structured interventions such as Computerized Cognitive Behavior Therapy (CCBT) have received the most attention over the last twenty years. Although we could not find any CCBT interventions utilizing NLG, positive results from their application in related areas (Barak et al. 2008) make them a prime candidate for future research. For example, the efficacy of Moodgym (Christensen, Griffiths and Jorm 2004), one of the pioneering tools for online CBT, showed that it was effective in reducing depression and dysfunctional thinking and in increasing understanding about CBT techniques. Since then many other CBT-based interventions have been developed and evaluated for the treatment of different mental health problems (Barak and Grohol 2011). Enough evidence for the effectiveness of online CBT has now been accumulated that in the UK computerized CBT is included in the 
public healthcare coverage (Kaltenthaler et al. 2006) and the market of commercial applications is booming (Aguilera and Muench 2012). NLP techniques could possibly be used to personalize the activities or to automatically process personal diaries.

A limitation of current CBT interventions that could be addressed with NLG systems is that they can only help when the user proactively goes to the website or installs the app. They cannot use any of the information users generate during their everyday life, such as Facebook posts, tweets, emails, etc. The NLP techniques described in Section 4 can be used to gather some of this information from these sources, which could be used to make inferences and generate appropriate interventions to be delivered privately through the same social media.

\subsubsection{Relational agents}

Relational agents (Bickmore and Picard 2005; Bohannon 2015) are tools where patients have conversations with a computer. The term relational aims to highlight a higher aim than similar conversational agents designed for question-answering or short dialogue situations (e.g. Apple's Siri). The research used to design relational agents feeds from psychology, sociolinguistics, communication and other behavioral sciences and focuses on how to build long-term relationships. The language used by the agents can vary in sophistication, going from multiple-choice questions, prerecorded texts or spoken language and most often include an NLG component.

Relational agents, and sometimes virtual reality (VR) environments, can interact and 'speak' to humans, i.e. they are Natural Language capable. To do this, they must understand spoken, or sometimes, written language, they must be able to manage a dialog (e.g. turn taking) and generate the output text/voice. Generally, the speech signal is converted to text using a speech recognition system, and the speech is generated from text using a text-to-speech system. Although details of this work is beyond the scope of the present discussion, Kenny et al. (2007) provide useful details on the architecture for one of these systems.

Briefly, dialogue systems work by understanding the users' dialogue move using the document classification techniques described in Section 4. These dialogue systems lookup for the closest match to the users' dialogue move (i.e. turn) and respond to it. If there is no statement close enough a default statement is used (e.g. 'sorry, I do not understand could you say that again').

For relational systems to work, they have to engage patients and build a good therapist-patient relationship (Okun and Kantrowitz 2014). It is believed that this relationship, and engagement in general, depend on the quality of the agents language, voice, metalinguistic and emotional expressions (e.g. face and body) (Bohannon 2015). Again this is similar to what has been done and evaluated in education. For example, D'Mello et al. (2011) develop affect-aware Intelligent Tutoring Systems, somewhat similar to relational agents, and show that when the system detects and responds to emotions it is better at promoting learning and engagement.

Maintaining engagement with any behavior change tool is challenging, even more so with agents that are expected to be used for a semester, a year or a whole 
life (Bickmore and Gruber 2010; Bickmore, Schulman and Yin 2010). Although short-term engagement is somewhat easier, it is not necessarily a good predictor of health outcomes (Bickmore et al. 2010). A way to study engagement with interventions involves using personal relationship research (Bickmore and Picard 2005) and medical psychology in general.

Bickmore and Gruber (2010) evaluated a number of systems used in health counseling and behavior change interventions. They found that the agents can be used in CBT interventions, can be engaging and help in behavior change, and they can also reduce cost or facilitate communication when human resources are scarce. The narrative is considered particularly important to the success of the intervention and can be designed to be engaging and possibly educational.

Some virtual agents use novel forms of data to personalize the dialogues. Help4Mood (Martínez-Miranda, Bresó and García-Gómez 2012b) is an interactive virtual agent aiming to help people recover from depression in their own homes. It uses subjective assessments, standard mood and depression questionnaires and diaries to personalize the conversations. An interesting feature of Help4Mood is that it also tracks aspects of behavior such as sleep and activity levels that can be used to shape the conversations. It is designed for use with other forms of counseling and therapy and the information collected can be provided to therapists.

Factors that influence the quality of relationships with an agent include the User Interface (Bickmore and Mauer 2006). For example, a text avatar (RincónNigro and Deng 2013), similar to a chatbox, would have different qualities than an embodied avatar. Some form of empathy in the avatar has been recognized as important and incorporated in several agents (Bickmore, Gruber and Picard 2005; Martínez-Miranda, Bresó and García-Gómez 2012a).

\subsection{Text messaging}

High-tech options like relational agents and virtual reality environments are not always the most appropriate due to cost, reach or because they cannot be incorporated into everyday life. Other media, such as text messaging (SMS) can be used to send feedback using a mixture of preprogrammed parts and individually tailored information (Bauer et al. 2003). For example, automated text messaging was used by Aguilera and Muñoz (2011) to support CBT in an adult, low-income population where smart phones and Internet access are not as common. It was aimed at increasing homework adherence, improving self-awareness and helping track patient progress by engaging patients through questions and simple activities. The small trial showed evidence that automated text messaging 'conversations' could be a low-cost approach to helping low-income populations.

Fathom (Dinakar et al. 2014) is a system to help counselors who provide help through SMS interventions (Crisis Text Line). Fathom uses topic models, a statistical modeling technique to track the evolution of themes in a conversation, in real time. The models were being built with 8,106 conversations held by 214 counselors. The evaluation did not yet include the topic maps with the full dataset but consisted of 
understanding the experiences of seven counselors using the system: they all found it most useful and had few feature requests.

A study to help patients with Bulimia Nervosa (Bauer et al. 2003) used canned and personalized messages. In this study, patients would send a weekly message to the system and receive automated feedback that contained a template and personalized segments. The pilot program provided evidence that it was well received and could be useful in aftercare treatment of patients. Regrettably effectiveness was not reported.

Academic evaluations of commercial tools like Buddyapp were not found. Buddyapp involves writing a diary and engaging with a self-help CBT-type intervention by sending text messages, so these are not included here.

\subsection{Online counseling}

Technology in this category - often referred as e-therapy - is used to mediate the interactions between patients and clinicians (e.g. Email, Skype) (Grohol 2004) and can replace a face-to-face session. The forms of communication or interaction can be more flexible for counseling and can be asynchronous (e.g. email, forums, etc.) or synchronous (e.g. chat, video conference, etc.).

Chatboxes are an interesting form of therapy. As shown by Dowling and Rickwood (2013) in a systematic review of online counseling via chat they can be effective in helping patients. For example, clients using the online chat service have shown positive attitude toward counseling (Finn and Bruce 2008). We could not find any recent study using NLP-enhanced chatboxes, and regard this as an important area that requires further evaluation.

\subsection{Online support groups and blogs}

Online mental health support groups have become increasingly popular since the late 1990s. They enable people in distress to find others with similar needs and problems, to share feelings and information, receive support, provide advice and develop a sense of community. For example, the feedback received by peer-supporters on social media can help individuals reflect on their thoughts, which offers a type of mental health intervention through the feedback loop (Hoyt and Pasupathi 2008; Grohol 2010). Online peer-support groups are effective in mental health and the degree to which a user engages with the group through posting new messages or replying to others, has shown to relate to the quality of the health outcome (Barak, Boneh, and Dolev-Cohen 2010). The review by Griffiths et al. (2009) focused on measuring the effectiveness of online support groups. They reviewed 31 papers (involving twenty-eight trials) and found that $62.5 \%$ reported a positive effect on depressive symptoms, although only $20 \%$ used control groups. Regrettably the coding did not include any technical features of the studies, such as use of NLP.

Moderator Assistant (Liu et al. 2013), described earlier, provides trained moderators the ability to identify people at risk and to respond with resources (e.g. link to mental health related information). These moderators help keep the community together and raise the value of conversations. Besides the triage system described in 
Section 5.5.1, Moderator Assistant can also automatically create draft interventions that moderators are expected to edit using NLG techniques (Hussain et al. 2015). These drafts can improve the efficiency and quality of the feedback provided. For example, they can use therapeutic language and focus on issues that reflect the ethos of the organization. If the organization wants to focus on certain illnesses or treatments it can develop specialized content.

\subsubsection{Triage systems}

Most research has used real data but largely neglected the translation of insights to real-life application of the diagnostic outcome. An understudied area is that of real use triage systems, used 'in the wild,' where individuals considered 'at risk' are identified in order to receive some form of assistance. We have already discussed studies by Choudhury and others who used Twitter (De Choudhury et al. 2013a) and Facebook (De Choudhury and Counts 2014) data and where recent mothers at risk of depression were identified, yet these studies did not evaluate how those people could be offered assistance, or whether this would even be acceptable.

For instance, a triage system that used social media data to alert people when someone in their network was depressed or suicidal was "Radar" created by the Good Samaritans in the United Kingdom (Horvitz and Mulligan 2015). The system quickly became an example of how difficult it is to use this data, even when it is for a noble cause, when the appropriate research has not been carried out as to how to provide feedback. The system used data from Facebook friends in ways they may not know about and this raised concerns about privacy. It also raised important issues around disclosure, such as who should find out that a person is not doing well or depressed. This information can be used by friends or foes, and even when it is by someone who wants to help, the person might not be the best qualified or might even get harmed herself - e.g. suicides occasionally happen in clusters of friends (Haw et al. 2013).

Another approach is for this type of technology to be used only by people who have been trained to help with mental health issues. For example, "Moderator Assistant" (Liu et al. 2013) is a triage system for moderators at ReachOut.com a mental health organization based in Australia. Some ReachOut.com users seek help by posting in discussion forums that have moderators who read the posts and where appropriate, respond. Their triage system aimed to help prioritize responses by identifying posts that needed urgent response together with information on the topic/issue. For the moderator, this might involve replying to the post, contacting the post author directly, and sometimes removing posts that do not comply with ReachOut.com policies. Given that this is often a complex decision, the triage tool also allows multiple moderators to communicate around a particular post.

\subsection{Summary}

- Text-driven psychological interventions are possibly the most common form of Internet intervention. 
- NLP techniques are rarely used as part of interventions.

- Psycho-educational and self-guided interventions have many similarities to learning technologies where NLP have been widely used. There is an opportunity for one area to learn from experiences in the other.

- Relational agents have been increasingly common, often in the form of embodied conversational agents or companions. The increased popularity of chatbots and automated messaging systems will possibly support growth in this line of research.

- Text messaging, often considered as a low-tech option is very useful, particularly in certain populations.

- Online counseling and peer support groups provide human-to-human communication where the computer mediation can add significant value through automatic triaging, summarization and personalization of the interventions.

\section{Conclusions}

The review was aimed to provide a taxonomy of how NLP has been used in mental health applications and potential future opportunities for its integration into online mental health tools. This application domain is highly interdisciplinary, with the technical aspects of building systems and the mental health challenges of helping those who most need it. A common problem is that the research literature in one area is often not known to researchers in the other which makes collaboration difficult. In fact, often these collaborations can be hindered by differences in the language, terminology and methodology. We covered three areas in which multidisciplinary teams could collaborate:

(1) data collection,

(2) processing or diagnosing and

(3) generation of automated mental health interventions.

Multidisciplinary teams have used data from Twitter, Facebook, blogs and other social media services to learn about people's behavior, emotions, social communications and more (Section 3). Most of the research has focused on English language, and more research is needed on other languages, particularly taking into account how cultural factors such as mental health views and stigma may influence the outcomes.

What we called 'labeling' (Section 4) encompasses triaging people at risk, the diagnosis of specific mental health conditions and even automatic labeling of suicide letters. There is probably other ways in which information extraction and text classification techniques can be used, but we have limited our discussion to those described in the mental health literature with a focus on depression and suicide. Although the techniques are similar to those used in other application domains, one has to be aware of the differences. For example, while classification accuracy in marketing is important, a false negative (i.e. erroneously not identifying an individual as belonging to a group) in marketing only means an opportunity is lost, while in mental health it could be more serious such as not identifying someone who is in 
need of help. The trade-offs between precision and recall will likely be different in these applications.

Although there has been great progress in the data collection and processing, there has been insufficient research on the uses of NLG in mental health interventions, with the notable exception being the use of relational agents.

\subsection{Research limitations}

This review is not an exhaustive compilation of all the work in NLP and mental health. The collaboration between psychologists and computing researchers is rapidly evolving, and new studies at the intersection of these fields are published regularly. For example, a recent special issue of Science Magazine dedicated to progress in AI had several mentions to this area (Bohannon 2015; Hirschberg and Manning 2015; Horvitz and Mulligan 2015).

Because of the character of the data, and the difficulty in maintaining the anonymity of those who write the texts (Horvitz and Mulligan 2015) ethical considerations are crucial. In fact, researchers should be aware of difficulty of applying methods common in computer science such as shared task competitions or data sharing. We have not covered the ethical implications of being able to identify people in need. This is an important area that requires a dedicated article. Once methods are developed to accurately process information data in mental health, a clear area that requires further research is how this information can be relayed sensitively and ethically back to participants.

\section{References}

Abbe, A., Grouin, C., Zweigenbaum, P., and Falissard, B. 2015. Text mining applications in psychiatry: a systematic literature review. International Journal of Methods in Psychiatric Research 25(2): 86-100.

Aguilera, A., and Muench, F. 2012. There's an App for that: information technology applications for cognitive behavioral practitioners. The Behavior Therapist/AABT 35(4): 65-73.

Aguilera, A., and Muñoz, R. F. 2011. Text messaging as an adjunct to CBT in low-income populations: a usability and feasibility pilot study. Professional Psychology: Research and Practice 42(6): 472-8.

American College Health Association 2009. National college health assessment spring 2008 reference group data report. Journal of American College Health 57, 477-88.

Armstrong, R., Hall, B. J., Doyle, J., and Waters, E. 2011. 'Scoping the scope' of a cochrane review. Journal of Public Health 33(1): 147-150.

Barak, A. 2007. Emotional support and suicide prevention through the internet: a field project report. Computers in Human Behavior 23(2): 971-984.

Barak, A., Boneh, O., and Dolev-Cohen, M. 2010. Factors underlying participants' gains in online support groups. In A. Blachnio, A. Przepiorka and T. Rowiński (eds.), Internet in psychological research, Warsaw, Poland: Cardinal Stefan Wyszyński University Press, pp. 13-47.

Barak, A., and Grohol, J. M. 2011. Current and future trends in internet-supported mental health interventions. Journal of Technology in Human Services 29(3): 155-196. 
Barak, A., Hen, L., Boniel-Nissim, M., and Shapira, N. 2008. A comprehensive review and a meta-analysis of the effectiveness of internet-based psychotherapeutic interventions. Journal of Technology in Human Services 26(2-4): 109-160.

Barak, A., and Miron, O. 2005. Writing characteristics of suicidal people on the Internet: a psychological investigation of emerging social environments. Suicide and Life-Threatening Behavior 35(5): 507-524.

Bauer, S., Percevic, R., Okon, E., Meermann, R. U., and Kordy, H. 2003. Use of text messaging in the aftercare of patients with bulimia nervosa. European Eating Disorders Review 11(3): 279-290.

Bental, D., and Cawsey, A. 2002. Personalized and adaptive systems for medical consumer applications. Communications of the ACM 45(5): 62-63.

Bental, D. S., Cawsey, A., and Jones, R. 1999. Patient information systems that tailor to the individual. Patient Education and Counseling 36, 171-180.

Bewick, B. M., Trusler, K., Barkham, M., Hill, A. J., Cahill, J., and Mulhern, B. 2008. The effectiveness of web-based interventions designed to decrease alcohol consumption-a systematic review. Preventive Medicine 47(1): 17-26.

Bickmore, T., and Gruber, A. 2010. Relational agents in clinical psychiatry. Harvard Review of Psychiatry 18(2): 119-130.

Bickmore, T., Gruber, A., and Picard, R. 2005. Establishing the computer-patient working alliance in automated health behavior change interventions. Patient Education and Counseling 59(1): 21-30.

Bickmore, T., and Mauer, D. 2006. Modalities for building relationships with handheld computer agents. In Proceedings of the CHI'06 Extended Abstracts on Human Factors in Computing Systems - CHI EA'06, New York, pp. 544-549.

Bickmore, T., Schulman, D., and Yin, L. 2010. Maintaining engagement in long-term interventions with relational agents. Applied Artificial Intelligence 24(6): 648-666.

Bickmore, T. W., and Picard, R. W. 2005. Establishing and maintaining longterm human-computer relationships. ACM Transactions on Computer-Human Interaction ( TOCHI) 12(2): 293-327.

Binstead, K., Cawsey, A., and Jones, R. 1995. Generating personalised information using the medical record. In Proceedings of Artificial Intelligence In Medicine, Berlin, New York, pp. 29-41.

Bohannon, J. 2015. The synthetic therapist. Science 349(6245): 250-251.

Bradley, M. M., and Lang, P. J. 1999. Affective norms for english words (anew): instruction manual and affective ratings. Technical Report C-1, The Center for Research in Psychophysiology, University of Florida.

Brew, C. 2016. Classifying reachout posts with a radial basis function svm. In Proceedings of the 3rd Workshop on Computational Linguistics and Clinical Psychology, San Diego, CA, USA, pp. 138-142.

Buchanan, B. G., Moore, J. D., Forsythe, D. E., Carenini, G., Ohlsson, S., and Banks, G. 1995. An intelligent interactive system for delivering individualized information to patients. Artificial Intelligence in Medicine 7(2): 117-154.

Calvo, R., and D'Mello, S. 2010. Affect detection: an interdisciplinary review of models, methods, and their applications. IEEE Transactions on Affective Computing 1(1): 18-37.

Calvo, R. A., Dinakar, K., Picard, R., and Maes, P. 2016. Computing in mental health. In Proceedings of the 2016 CHI Conference Extended Abstracts on Human Factors in Computing Systems, Santa Clara, California, USA, pp. 3438-3445.

Calvo, R. A., and Kim, S. 2013. Emotions in text: dimensional and categorical models. Computational Intelligence 29(3): 527-543.

Calvo, R. A., Peters, D., and D'Mello, S. 2015. When technologies manipulate our emotions. Communications of the ACM 58(11): 41-42.

Cherry, C., Mohammad, S. M., and De Bruijn, B. 2012. Binary classifiers and latent sequence models for emotion detection in suicide notes. Biomedical Informatics Insights 5(Suppl 1): $147-154$. 
Christensen, H., Batterham, P., Mackinnon, A., Griffiths, K. M., Hehir, K. K., Kenardy, J., Gosling, J., and Bennett, K. 2014. Prevention of generalized anxiety disorder using a web intervention, iChill: randomized controlled trial. Journal of Medical Internet Research 16(9): e199.

Christensen, H., Griffiths, K. M., and Jorm, A. F. 2004. Delivering interventions for depression by using the internet: randomised controlled trial. BMJ 328(7434): 265-268.

Chung, C., and Pennebaker, J. W. 2007. The psychological function of function words. In K. Fiedler (ed.), Social communication, New York: Psychology Press, pp. 343-359.

Coch, J. 1996. Evaluating and comparing three text-production techniques. In Proceedings of the 16th Conference on Computational Linguistics, vol. 1. Stroudsburg, PA, USA, pp. 249254.

Coppersmith, G., Dredze, M., Harman, C., Hollingshead, K., and Mitchell, M. 2015. Clpsych 2015 shared task: depression and ptsd on twitter. In Proceedings of the 2nd Workshop on Computational Linguistics and Clinical Psychology: From Linguistic Signal to Clinical Reality, Denver, Colorado, pp. 31-39.

Coviello, L., Sohn, Y., Kramer, A. D., Marlow, C., Franceschetti, M., Christakis, N. A., and Fowler, J. H. 2014. Detecting emotional contagion in massive social networks. PloS One 9(3): e90315.

De Carolis, B., de Rosis, F., Grasso, F., Rossiello, A., Berry, D. C., and Gillie, T. 1996. Generating recipient-centered explanations about drug prescription. Artificial Intelligence in Medicine 8(2): 123-145.

De Choudhury, M., and Counts, S. 2014. Characterizing and predicting postpartum depression from shared facebook data. Proceedings of the 17th ACM Conference on Computer Supported Cooperative Work and Social Computing, New York, NY, USA, pp. 626-638.

De Choudhury, M., Counts, S., and Horvitz, E. 2013a. Predicting postpartum changes in emotion and behavior via social media. In Proceedings of the SIGCHI Conference on Human Factors in Computing Systems, New York, NY, USA, pp. 3267-3276.

De Choudhury, M., Counts, S., and Horvitz, E. 2013b. Social Media As a Measurement Tool of Depression in Populations. In Proceedings of the 5th Annual ACM Web Science Conference (WebSci '13). New York, NY, USA: ACM, pp. 47-56.

De Choudhury, M., and De, S. 2014. Mental health discourse on reddit: self-disclosure, social support, and anonymity. In The International AAAI Conference on Weblogs and Social Media (ICWSM), Ann Arbor, MI, USA, pp. 71-80.

De Choudhury, M., Gamon, M., Counts, S., and Horvitz, E. 2013. Predicting depression via social media. In The International AAAI Conference on Weblogs and Social Media (ICWSM), Boston, USA, pp. 128-137.

DiMarco, C., Covvey, H., Cowan, D., DiCiccio, V., Hovy, E., Lipa, J., and Mulholland, D. 2007. The development of a natural language generation system for personalized e-health information. In Medinfo 2007: Proceedings of the 12th World Congress on Health (Medical) Informatics; Building Sustainable Health Systems, Amsterdam, Netherlands, pp. 2339-2344.

Dinakar, K., Chaney, A. J. B., Lieberman, H., and Blei, D. M. 2014. Real-time topic models for crisis counseling. In Proceedings of the 20th ACM Conference on Knowledge Discovery and Data Mining, Data Science for the Social Good Workshop, New York, USA.

D'Mello, S. K., Lehman, B., and Graesser, A. 2011. A Motivationally Supportive AffectSensitive AutoTutor. Inbook. In R. A. Calvo and S. K. D’Mello (eds.), New Perspectives on Affect and Learning Technologies, Springer New York, pp. 113-126.

Dockrey, M. 2007. Emulating mental state in natural language generation systems. Technical Report, University of British Columbia.

Dodds, P. S., Harris, K. D., Kloumann, I. M., Bliss, C. A., and Danforth, C. M. 2011. Temporal patterns of happiness and information in a global social network: hedonometrics and twitter. PloS One 6(12): e26752.

Donker, T., Griffiths, K. M., Cuijpers, P., and Christensen, H. 2009. Psychoeducation for depression, anxiety and psychological distress: a meta-analysis. BMC Medicine 7(1): e79. 
Dowling, M., and Rickwood, D. 2013. Online counseling and therapy for mental health problems: a systematic review of individual synchronous interventions using chat. Journal of Technology in Human Services 31(1): 1-21.

Durkheim, E. 1897. Suicide: A Study in Sociology. [1951] Glencoe, Illinois: Free Press.

Eysenbach, G., Powell, J., Englesakis, M., Rizo, C., and Stern, A. 2004. Health related virtual communities and electronic support groups: systematic review of the effects of online peer to peer interactions. BMJ 328(7449): 1166-1172.

Finn, J., and Bruce, S. 2008. The LivePerson model for delivery of etherapy services: a case study. Journal of Technology in Human Services 26(2-4): 282-309.

Forman, G. 2003. An extensive empirical study of feature selection metrics for text classification. The Journal of machine learning research 3; 1289-1305.

Gatt, A., Portet, F., Reiter, E., Hunter, J., Mahamood, S., Moncur, W., and Sripada, S. 2009. From data to text in the neonatal intensive care unit: using NLG technology for decision support and information management. A.I. Communications 22(3): 153-186.

Golder, S., and Macy, M. 2011. Diurnal and seasonal mood vary with work, sleep, and daylength across diverse cultures. Science 333(6051): 1878-1881.

Griffiths, M. K., Calear, L. A., and Banfield, M. 2009. Systematic review on internet support groups (ISGs) and depression (1): do ISGs reduce depressive symptoms? Journal of Medical Internet Research 11(3): e40.

Grohol, J. M. 2004. Online counseling: a historical perspective. In R. Kraus, G. Stricker, and C. Speyer (eds.), Online Counseling: A Handbook for Mental Health Professionals, San Diego, CA: Elsevier Academic Press, pp. 51-68.

Grohol, J. M. 2010. Using websites, blogs and wikis in mental health. In K. Anthony, D. A. N. Nagel, and S. Goss (eds.), The use of technology in mental health applications ethics and practice, Springfield, IL: Charles C. Thomas, pp. 68-75.

Haw, C., Hawton, K., Niedzwiedz, C., and Platt, S. 2013. Suicide clusters: a review of risk factors and mechanisms. Suicide and Life-Threatening Behavior 43(1): 97-108.

He, Q., Veldkamp, B. P., Glas, C. A., and de Vries, T. 2015. Automated assessment of patients' self-narratives for posttraumatic stress disorder screening using natural language processing and text mining. Assessment 0(0): 1-16. SAGE Publications.

Hirschberg, J., and Manning, C. D. 2015. Advances in natural language processing. Science 349(6245): 261-266.

Homan, C. M., Johar, R., Liu, T., Lytle, M., Silenzio, V., and Alm, C. O. 2014. Toward macro-insights for suicide prevention: analyzing fine-grained distress at scale. In $A C L$ 2014, Baltimore, MD, USA, pp. 107-117.

Homan, C. M., Lu, N., Tuurmcrochesteredu, N. L. X., Lytle, M. C., Lytle, M., Rochester, U., Silenzio, V. M. B., and Silenzio, V. 2014a. Social structure and depression in TrevorSpace. In Proceedings of the 17th ACM Conference on Computer Supported Cooperative Work and Social Computing - CSCW'14, Baltimore, MD, USA, pp. 615-624.

Homan, C. M., Lu, N., Tuurmcrochesteredu, N. L. X., Lytle, M. C., Lytle, M., Rochester, U., Silenzio, V. M. B., and Silenzio, V. 2014b. Social structure and depression in TrevorSpace. In Proceedings of the 17th ACM Conference on Computer Supported Cooperative Work and Social Computing - CSCW'14, Baltimore, MD, USA, pp. 615-624.

Horvitz, E., and Mulligan, D. 2015. Data, privacy, and the greater good. Science 349(6245): 253-255.

Hoyt, T., and Pasupathi, M. 2008. Blogging about trauma: linguistic measures of apparent recovery. E-Journal of Applied Psychology 4(2): 56-62.

Hussain, M. S., Calvo, R. A., Ellis, L., Li, J., Ospina-Pinillos, L., Davenport, T., and Hickie, I. 2015. Nlg-based moderator response generator to support mental health. In Proceedings of the 33rd Annual ACM Conference Extended Abstracts on Human Factors in Computing Systems, Seoul, Republic of Korea, pp. 1385-1390.

Jones, R., Pearson, J., McGregor, S., Cawsey, A. J., Barrett, A., Craig, N., Atkinson, J. M., Gilmour, W. H., and McEwen, J. 1999. Randomised trial of personalised computer based information for cancer patients. BMJ 319(7219): 1241-1247. 
Kaltenthaler, E., Brazier, J., De Nigris, E., Tumur, I., Ferriter, M., Beverley, C., Parry, G., Rooney, G., and Sutcliffe, P. 2006. Computerised cognitive behaviour therapy for depression and anxiety update: a systematic review and economic evaluation. Health Technology Assessment 10(33): 1-186.

Kenny, P., Parsons, T. D., Gratch, J., Leuski, A., and Rizzo, A. A. 2007. Virtual patients for clinical therapist skills training. In Intelligent Virtual Agents, Springer-Verlag Berlin Heidelberg, pp. 197-210.

Kim, S. M., Wang, Y., Wan, S., and Paris, C. 2016. Data61-csiro systems at the clpsych 2016 shared task. In Proceedings of the 3rd Workshop on Computational Linguistics and Clinical Psychology, San Diego, CA, USA, pp. 128-132.

Kotsiantis, S. 2007. Supervised machine learning: a review of classification techniques. Informatica 31; 249-268.

Kramer, A. D. 2010. An unobtrusive behavioral model of gross national happiness. In Proceedings of the SIGCHI Conference on Human Factors in Computing Systems, Atlanta, GA, USA, pp. 287-290.

Kramer, A. D., Guillory, J. E., and Hancock, J. T. 2014. Experimental evidence of massivescale emotional contagion through social networks. Proceedings of the National Academy of Sciences 111(24): 8788-8790.

Larsen, M. E., Boonstra, T. W., Batterham, P. J., O’Dea, B., Paris, C., and Christensen, H. 2015. We feel: mapping emotion on twitter. IEEE Journal of Biomedical and Health Informatics 19(4): 1246-1252.

Lawless, N., and Lucas, R. 2011. Predictors of regional well-being: a county level analysis. Social Indicators Research 101(3): 341-357.

Li, A., Huang, X., Hao, B., O’Dea, B., Christensen, H., and Zhu, T. 2015. Attitudes towards suicide attempts broadcast on social media: an exploratory study of chinese microblogs. PeerJ 3: e1209.

Liu, M., Calvo, R. A., Davenport, T., and Hickie, I. 2013. Moderator assistant: helping those who help via online mental health support groups. In Joint Workshop on Smart Health and Social Therapies, OzChi, pp. 1-4.

Luyckx, K., Vaassen, F., Peersman, C., and Daelemans, W. 2012. Fine-grained emotion detection in suicide notes: a thresholding approach to multi-label classification. Biomedical Informatics Insights 5(Suppl 1): 61-69.

Malmasi, S., Zampieri, M., and Dras, M. 2016. Predicting post severity in mental health forums. In Proceedings of the 3rd Workshop on Computational Linguistics and Clinical Psychology, San Diego, CA, USA, pp. 133-137.

Martínez-Miranda, J., Bresó, A., and García-Gómez, J. M. 2012a. Modelling therapeutic empathy in a virtual agent to support the remote treatment of major depression. In ICAART (2), Vilamoura, Algarve, Portugal, pp. 264-269.

Martínez-Miranda, J., Bresó, A., and García-Gómez, J. M. 2012b. The construction of a cognitive-emotional module for the Help4Mood's virtual agent. In Proceedings of 1st Workshop on Information and Communication Technologies Applied to Mental Health, Valencia, Spain, pp. 34-39.

Masuda, N., Kurahashi, I., and Onari, H. 2013. Suicide ideation of individuals in online social networks. PloS One 8(4): e62262.

McCart, J. A., Finch, D. K., Jarman, J., Hickling, E., Lind, J. D., Richardson, M. R., Berndt, D. J., and Luther, S. L. 2012. Using ensemble models to classify the sentiment expressed in suicide notes. Biomedical Informatics Insights 5(Suppl 1): 77-85.

Miller, G., Beckwith, R., Fellbaum, C., Gross, D., and Miller, K. 1990. Introduction to wordnet: an on-line lexical database. Journal of Lexicography 3, 235-244.

Milne, D. N., Pink, G., Hachey, B., and Calvo, R. A. 2016. Clpsych 2016 shared task: triaging content in online peer-support forums. In Proceedings of the 3rd Workshop on Computational Linguistics and Clinical Psychology, San Diego, CA, USA, pp. 118-127. 
Mitchell, L., Frank, M. R., Harris, K. D., Dodds, P. S., and Danforth, C. M. 2013. The geography of happiness: connecting twitter sentiment and expression, demographics, and objective characteristics of place. PloS One 8(5): e64417.

Moreno, M., and Jelenchick, L. 2011. Feeling bad on Facebook: depression disclosures by college students on a social networking site. Depression and Anxiety 28: 447-455.

Myung, S.-K., McDonnell, D. D., Kazinets, G., Seo, H. G., and Moskowitz, J. M. 2009. Effects of Web-and computer-based smoking cessation programs: meta-analysis of randomized controlled trials. Archives of Internal Medicine 169(10): 929-937.

Neve, M., Morgan, P. J., Jones, P. R., and Collins, C. E. 2010. Effectiveness of web-based interventions in achieving weight loss and weight loss maintenance in overweight and obese adults: a systematic review with meta-analysis. Obesity Reviews 11(4): 306-321.

Nguyen, T., Phung, D., Dao, B., Venkatesh, S., and Berk, M. 2014. Affective and content analysis of online depression communities. IEEE Transactions on Affective Computing 5(3): 217-226.

Nigam, K., McCallum, A. K., Thrun, S., and Mitchell, T. 2000. Text classification from labeled and unlabeled documents using EM. Machine Learning 39(2-3): 103-134.

O'Dea, B., and Campbell, A. 2010. Healthy connections: online social networks and their potential for peer support. Studies in Health Technology and Informatics 168: 133-140.

O’Dea, B., Wan, S., Batterham, P. J., Calear, A. L., Paris, C., and Christensen, H. 2015. Detecting suicidality on Twitter. Internet Interventions 2(2): 183-188.

Okun, B., and Kantrowitz, R. 2014. Effective Helping: Interviewing and Counseling Techniques. Cengage Learning. Toronto, Canda: Nelson Education.

Owen, J. E., Klapow, J. C., Roth, D. L., Shuster, J. L., Bellis, J., Meredith, R., and Tucker, D. C. 2005. Randomized pilot of a self-guided internet coping group for women with early-stage breast cancer. Annals of Behavioral Medicine 30(1): 54-64.

Paul, M., and Dredze, M. 2011. You are what you tweet: Analyzing twitter for public health. In Proceedings of the 5th International AAAI Conference on Weblogs and Social Media, Barcelona, Spain, pp. 265-272.

Pedersen, T. 2015. Screening twitter users for depression and ptsd with lexical decision lists. In Proceedings of the 2nd Workshop on Computational Linguistics and Clinical Psychology: From Linguistic Signal to Clinical Reality, Denver, Colorado, pp. 46-53.

Peek, N., Combi, C., Marin, R., and Bellazzi, R. 2015. Artificial intelligence in medicine thirty years of artificial intelligence in medicine ( AIME ) conferences: a review of research themes. Artificial Intelligence In Medicine 65(1): 61-73.

Pennebaker, J., Kiecolt-Glaser, J., and Glaser, R. 1988. Disclosure of traumas and immune function: health implications for psychotherapy. Journal of Consulting and Clinical Psychology 56(2): 239-245.

Pennebaker, J. W. 2011. The Secret Life of Pronouns: How Our Words Reflect Who We Are. New York, NY: Bloomsbury Press.

Pennebaker, J. W., Boyd, R. L., Jordan, K., and Blackburn, K. 2015. The development and psychometric properties of liwc2015. UT Faculty/Researcher Works.

Pennebaker, J. W., and Chung, C. K. 2007. Expressive writing, emotional upheavals, and health. Handbook of Health Psychology, Oxford University Press, USA, pp. 263-284.

Pestian, J., Nasrallah, H., Matykiewicz, P., Bennett, A., and Leenaars, A. 2010. Suicide note classification using natural language processing: a content analysis. Biomedical Informatics Insights 2010(3): 19-28.

Pestian, J. P., Matykiewicz, P., Linn-Gust, M., South, B., Uzuner, O., Wiebe, J., Cohen, K. B., Hurdle, J., and Brew, C. 2012. Sentiment analysis of suicide notes: a shared task. Biomedical Informatics Insights 5(Suppl 1): 3-16.

Pistrang, N., Barker, C., and Humphreys, K. 2008. Mutual help groups for mental health problems: a review of effectiveness studies. American Journal of Community Psychology 42(1-2): 110-121. 
Preoţiuc-Pietro, D., Sap, M., Schwartz, H. A., and Ungar, L. 2015. Mental illness detection at the world well-being project for the clpsych 2015 shared task. In Proceedings of the 2nd Workshop on Computational Linguistics and Clinical Psychology: From Linguistic Signal to Clinical Reality, Denver, Colorado, pp. 40-45.

Reiter, E., and Dale, R. 2000. Building Natural Language Generation Systems. Boston: MIT Press.

Reiter, E., Robertson, R., and Osman, L. M. 2003. Lessons from a failure: generating tailored smoking cessation letters. Artificial Intelligence 144(1): 41-58.

Resnik, P., Armstrong, W., Claudino, L., and Nguyen, T. 2015. The university of maryland clpsych 2015 shared task system. In Proceedings of the 2nd Workshop on Computational Linguistics and Clinical Psychology: From Linguistic Signal to Clinical Reality, Denver, Colorado, pp. 54-60.

Resnik, P., Resnik, R., and Mitchell, M. (eds.) 2014. Proceedings of the Workshop on Computational Linguistics and Clinical Psychology: From Linguistic Signal to Clinical Reality. Baltimore, Maryland, USA: Association for Computational Linguistics, pp. 1125.

Rincón-Nigro, M., and Deng, Z. 2013. A text-driven conversational avatar interface for instant messaging on mobile devices. IEEE Transactions on Human-Machine Systems 43(3): $328-332$.

Ritterband, L. M., Gonder-Frederick, L. A., Cox, D. J., Clifton, A. D., West, R. W., and Borowitz, S. M. 2003. Internet interventions: in review, in use, and into the future. Professional Psychology: Research and Practice 34(5): 527-534.

Riva, G., Calvo, R. A., and Lisetti, C. 2014. Cyberpsychology and affective computing. In R. Calvo, S. D’Mello, J. Gratch, and A. Kappas (eds.), Handbook of Affective Computing, pp. 547-558. New York: Oxford University Press.

Sadilek, A., Homan, C., Lasecki, W., Silenzio, V., and Kautz, H. 2013. Modeling fine-grained dynamics of mood at scale. In WSDM, Rome, Italy, pp. 3-6.

Schwartz, H. A., Eichstaedt, J. C., Margaret L. Kern, L., Dziurzynski, M. A., Park, G. J., Lakshmikanth, S. K., Jha, S., Seligman, M. E. P., and Ungar, L. 2013. Characterizing geographic variation in well-being using tweets. In Proceedings of the 7th International AAAI Conference on Weblogs and Social Media, Cambridge, Massachusetts, USA, pp. 583591.

Sebastiani, F. 2002. Machine learning in automated text categorization. ACM Comput. Surv. 34(1): 1-47.

Shneidman, E., and Farberow, N. (eds.) 1957. Clues to Suicide. New York: Harper and Row.

Signorini, A., Segre, A. M., and Polgreen, P. M. 2011. The use of Twitter to track levels of disease activity and public concern in the US during the influenza A H1N1 pandemic. PloS One 6(5): e19467.

Spasić, I., Burnap, P., Greenwood, M., and Arribas-Ayllon, M. 2012. A naïve Bayes Approach to classifying Topics in suicide notes. Biomedical Informatics Insights 5(Suppl 1): 87-97.

Spek, V., Cuijpers, P. I. M., Nyklícek, I., Riper, H., Keyzer, J., and Pop, V. 2007. Internetbased cognitive behaviour therapy for symptoms of depression and anxiety: a meta-analysis. Psychological Medicine 37(03): 319-328.

Strapparava, C., and Mihalcea, R. 2007. Semeval-2007 task 14: affective text. In Proceedings of the 4th International Workshop on Semantic Evaluations, Prague, Czech Republic, pp. 70-74.

Strapparava, C., and Mihalcea, R. 2008. Learning to identify emotions in text. In Proceedings of the 2008 ACM Symposium on Applied Computing, Fortaleza, Ceara, Brazil, pp. 1556-1560.

Strapparava, C., and Mihalcea, R. 2014. Affect detection in texts. In R. A. Calvo, S. D’Mello, J. Gratch, and A. Kappas (eds.), The Oxford Handbook of Affective Computing, Chapter 13, pp. 184-203. New York: Oxford University Press.

Strapparava, C., and Valitutti, A. 2004. WordNet-Affect: an affective extension of WordNet. In LREC 2004 - 4th IInternational Conference on Language Resources and Evaluation, vol. 4, Lisbon, pp. 1083-1086. 
Tausczik, Y. R., and Pennebaker., J. W. 2010. The psychological meaning of words: LIWC and computerized text analysis methods. Journal of Language and Social Psychology 29(1): $24-54$.

Weizenbaum, J. 1966. ELIZA-a computer program for the study of natural language communication between man and machine. Communications of the ACM 9(1): 36-45.

Witten, I. H., and Frank, E. 2005. Data Mining: Practical Machine Learning Tools and Techniques. San Francisco, USA: Morgan Kaufmann.

Xu, Y., Wang, Y., Liu, J., Tu, Z., Sun, J.-T., Tsujii, J., and Chang, E. 2012. Suicide note sentiment classification: a supervised approach augmented by web data. Biomedical Informatics Insights 5(Suppl 1): 31-41.

Yang, H., Willis, A., De Roeck, A., and Nuseibeh, B. 2012. A hybrid model for automatic emotion recognition in suicide notes. Biomedical Informatics Insights 5(Suppl 1): 17-30.

Yu, N., Kübler, S., Herring, J., Hsu, Y.-Y., Israel, R., and Smiley, C. 2012. LASSA: emotion detection via information fusion. Biomedical Informatics Insights 5(Suppl 1): 71-76. 